\title{
Evaluación de la experiencia de uso de textos literarios como herramienta de enseñanza en cursos de introducción a la epistemología y metodología de la investigación social ${ }^{1}$
}

\section{Evaluating the experience of using literary works as a teaching tool in introductory courses to epistemology and methodology of social research}

ISSN 1510-2432 - ISSN 1688-9304 (en línea) - DOI: https://doi.org/10.18861/cied.2018.9.2.2853

Marcos Baudean

Master en Políticas Públicas, Universidad ORT Uruguay. Sociólogo, Universidad de la República (Uruguay). Investigador independiente. Ex jefe, departamento de Investigación y desarrollo, Mercoplus Latin America. Catedrático Asociado de Metodología de la Investigación, Facultad de Administración y Ciencias Sociales, Universidad ORT Uruguay.

Fanny Rudnitzky

Licenciada en Sociología, Universidad de la República (Uruguay). Profesora de Metodología de Investigación, Facultad de Administración y Ciencias Sociales, Universidad ORT Uruguay.

Fecha de recibido: 28/12/2017

Fecha de aceptado: 23/06/2018

\section{Resumen}

El presente trabajo señala las potencialidades del uso de narraciones literarias en la enseñanza de temáticas epistemológicas y metodológicas en cursos introductorios de Metodología de Investigación Social. Esta cuestión se abordará mediante la enumeración de algunas obras literarias que abarcan temas de relevancia para la reflexión metodológica o epistemológica y la ejemplificación de diferentes dinámicas de enseñanza mediante las cuales puede trabajarse el vínculo entre estas obras y conceptos de interés en el marco de cursos iniciales de formación de grado en Metodología de Investigación Social. Por último, se presentan resultados de una encuesta destinada a evaluar la herramienta por parte de la población estudiantil.

Palabras clave: enseñanza de metodología de investigación, enseñanza de epistemología, uso de textos literarios en la enseñanza de ciencias sociales, enseñanza universitaria, actividades para el aula.

\footnotetext{
Abstract

The document points out the benefits of using literary works in the teaching of epistemological and methodological topics in introductory courses on Social Research Methodology. In the article, some literary works that deal with topics of relevance for methodological or epistemological reflections are listed and described. Different teaching dynamics are presented and exemplified to show how to link literary works to epistemological or methodological concepts. In addition, the results of a survey designed to evaluate the tool by the student population are presented.

Keywords: Teaching of research methodology, teaching of epistemology, use of literary works in the teaching of social sciences, college teaching, classroom activities
} 


\section{Introducción}

En 1963 Lewis Coser publicaba Sociology through literature (Coser, 1972). Esta obra estaba organizada en forma de manual introductorio a la teoría sociológica, pero cada capítulo incluía una selección de textos literarios de diversos géneros (cuento corto, novela, teatro, poesía) para ilustrar los grandes conceptos de la teoría sociológica. El objetivo de Coser era potenciar la enseñanza de la sociología a través de material ilustrativo tomado de la literatura. Dicha estructura se fundamentaba en las dificultades de los estudiantes de grado (sobre todo en los primeros años) con respecto a las abstracciones de la teoría sociológica, abstracciones difíciles de relacionar con el mundo social directamente experimentado por los estudiantes.

Existe un problema similar con la enseñanza de la epistemología y la metodología de la investigación en los cursos de los primeros años del grado, pero con el agravante de que los temas epistemológicos tienden a cuestionar las verdades más fuertemente incorporadas por los estudiantes tales como las siguientes: ¿Cuándo podemos estar seguros de conocer algo? ¿Existe el mundo exterior a mi mente? ¿Es independiente de mí? Por su parte, los temas metodológicos requieren dudar, formalizar y argumentar, y esto hace que la investigación sea un camino de conocimiento en el que las decisiones de cómo avanzar deben estar fundamentadas. Los elementos señalados son, en general, ajenos a las experiencias de conocimiento previas de los estudiantes de grado.

El uso de textos literarios se ha popularizado desde la publicación del libro de Coser como una alternativa en la enseñanza de la sociología². La novedad de este artículo es el uso de textos literarios en la enseñanza de la metodología de la investigación.

En el presente artículo se presenta

1. Una lista ilustrativa de temáticas epistemológicas y metodológicas seguida de textos literarios empleados para trabajar con ellas.

2. Tres dinámicas para utilizar textos literarios en el aula o fuera de ella, junto con una serie de recomendaciones para la manipulación de textos y dinámicas, así como para la planificación del uso de relatos en el programa de la materia.

3. Los resultados de una evaluación realizada por estudiantes acerca del uso de textos literarios dentro de los cursos de metodología y epistemología a los que asistieron.

\section{La discusión: el uso de textos literarios para la enseñanza de las ciencias sociales}

El texto de Coser (1972) es señalado en diversas publicaciones como el primero en identificar el valor pedagógico de utilizar fuentes literarias para ilustrar conceptos sociológicos. Posteriormente, varios autores sistematizaron sus propias experiencias con respecto al uso de textos literarios en el marco de cursos de introducción a la sociología (Castellano, DeAngelis \& Clark-Ibáñez, 2008; Laz, 1996; Hegtvedt, 1991; Hill, 1987; Sullivan, 1982). Pese a ello son escasas las referencias al uso de este tipo de materiales para la enseñanza de epistemología y metodología (Collier, 2011).

A continuación se presenta una revisión de la literatura que muestra los principales argumentos que fundamentan el uso de textos literarios, dinámicas de clase para su uso y evaluación de resultados obtenidos. 
Principales argumentos para el uso de textos literarios

Un primer argumento consiste en que las piezas literarias pueden oficiar de puente entre las experiencias cotidianas de los estudiantes y el conocimiento generado por ellas, y la abstracción de la teoría sociológica.

The major problem in introductory sociology is the enormous gap between abstract sociological concepts (e.g., "deviance", "alienation", "system", indeed, "sociological perspective" itself) and the set of actual subjective - yet no less "social" —experiences of the student himself. As the responses suggest, literature provides a third, intermediary level of understanding: it describes experiences which are "outside" the student and not his own, allowing him to emphasize and generalize beyond his personal consciousness (Jones, 1975, pág. 195) ${ }^{3}$.

Generar un punto de partida común para todos los estudiantes a partir de la alusión a una obra literaria también reporta una ventaja a la hora de lidiar con grupos de sujetos provenientes de contextos diversos y con intereses disímiles (Sullivan, 1982, pág. 110). Otro argumento esgrimido a favor de la incorporación de materiales literarios en la enseñanza de la sociología es que su uso suele generar mayor motivación en los estudiantes. Esto se debe a que es un material fácilmente accesible y que suele estar acompañado de propuestas de trabajo y ejercicios que rompen con la tradicional lógica expositiva del docente (Castellano, DeAngelis \& Clark-Ibáñez, 2008, pág. 240).

La inclusión de material literario en los cursos introductorios de sociología suele realizarse en dos modalidades complementarias. Por un lado, en tanto que describen situaciones de la vida social estos materiales resultan de utilidad para ilustrar conceptos sociológicos. Por otro lado, las obras literarias también proveen multiplicidad de escenarios para ejercitar la aplicación de estos conceptos en contextos diversos (Coser, 1972; Castellano, DeAngelis \& Clark-Ibáñez, 2008; Hegtvedt, 1991; Sullivan, 1982).

Incorporado de esta forma, el material literario aporta no solo a la comprensión e incorporación de un set de conceptos relevantes para la disciplina en cuestión, sino que promueve también el desarrollo de ciertas habilidades o competencias en los estudiantes, alentando el pensamiento crítico, las habilidades analíticas (Castellano, DeAngelis \& ClarkIbáñez, 2008), el pensamiento creativo, la capacidad de resolución de problemas (Laz, 1996), el razonamiento analógico y la capacidad de aplicación conceptual (Sullivan, 1982).

\section{Dinámicas de clase utilizadas}

En la literatura reseñada se destacan algunos elementos comunes en el uso de los materiales literarios. En primer lugar, este tipo de materiales suele estar acompañado de una bibliografía propiamente sociológica que provee las herramientas conceptuales que permiten el análisis de los textos de ficción (Castellano, DeAngelis \& Clark-lbáñez, 2008; Sullivan, 1982). En segundo lugar, se destaca la generación de dinámicas que permitan discutir los contenidos del texto y orientar a los estudiantes para asegurar una correcta comprensión de los sucesos allí narrados. Estas dinámicas pueden consistir en la discusión de la trama de la obra literaria en sesiones colectivas (Laz, 1996) o en la puesta a disposición de una guía de preguntas que oriente la lectura de la obra (Castellano, DeAngelis \& ClarkIbáñez, 2008; Hegtvedt, 1991; Sullivan, 1982).

La discusión en clase y las guías de preguntas que se brindan a los estudiantes también se utilizan como insumo para avanzar, no ya en la comprensión de la trama de la obra sino en su interpretación a partir de una mirada sociológica. Las modalidades propuestas para resolver la consigna pautada pueden variar. Es posible presentar ejercicios individuales 
o grupales (de trabajo en subgrupos en una clase) y ejercicios en clase o como tarea domiciliaria (Castellano, DeAngelis \& Clark-Ibáñez, 2008; Laz, 1996; Hegtvedt, 1991; Sullivan, 1982). También se destaca la incorporación de ejercicios que incluyan la lectura e interpretación de fragmentos de obras literarias no trabajados previamente en el marco de evaluaciones presenciales del curso (Sullivan, 1982).

\section{Resultados obtenidos}

En la literatura estudiada es poco frecuente encontrar evaluación de resultados. Los estudios que sí reportan resultados se centran en la percepción de los estudiantes sobre el curso y los materiales empleados, y registran valoraciones favorables.

Castellano et al. aplicaron un cuestionario a estudiantes que habían participado en sus cursos. Los ítems evaluados fueron los siguientes:

El uso de textos no tradicionales contribuyó a estimular el interés en la materia;

Recomendaría esta metodología de trabajo para otros cursos;

- Los textos contribuyeron a una mejor comprensión de los conceptos sociológicos; y

- Los textos me ayudaron a entender cómo aplicar los conceptos sociológicos.

- Las opciones de respuesta fueron:

- De acuerdo,

- Neutralidad,

- Desacuerdo.

En todos los casos la opción más escogida fue De acuerdo (Castellano, DeAngelis \& ClarkIbáñez, 2008).

Jones evalúa su curso introductorio a la sociología valiéndose de las respuestas a un cuestionario de preguntas abiertas y cerradas, así como de entrevistas en profundidad realizadas a los estudiantes. A partir de estas técnicas se propone evaluar la valoración de los estudiantes del método aplicado y los aportes específicos de introducir este tipo de lecturas. Con relación a la primera dimensión los resultados son concluyentes. El 91\% de los estudiantes encuestados se manifiesta de acuerdo o muy de acuerdo con que la enseñanza de la sociología a través de la literatura es un buen método. Dada la ausencia de un grupo de control no es posible extraer resultados concluyentes en lo que se refiere al aporte específico de este tipo de metodología con relación a la metodología tradicional. No obstante, las respuestas al cuestionario y a las entrevistas en profundidad muestran indicios del potencial de aplicar este tipo de técnicas (Jones, 1975).

Finalmente, Sullivan compara los resultados obtenidos en pruebas nacionales estandarizadas por los estudiantes que tomaron su curso con los resultados obtenidos por grupos con similares características que tomaron cursos estándar. Dicha comparación registró mejores resultados para los primeros. Adicionalmente, en evaluaciones anónimas el $83 \%$ de los estudiantes del curso de Sullivan declara preferir el abordaje basado en el uso de textos literarios que el abordaje tradicional que no incorpora este tipo de materiales (Sullivan, 1982). 


\section{Relatos literarios utilizados en la enseñanza de epistemología y metodología}

En los cursos introductorios a Metodología de la Investigación pueden utilizarse relatos y fragmentos literarios con dos grandes finalidades. Una es que los estudiantes posean una visión general de los desafíos cognitivos que enfrenta un investigador. La otra se refiere el abordaje de temáticas específicas (introducir una temática nueva, promover una discusión sobre un tema y poner en práctica conceptos trabajados previamente).

En lo que sigue se presentan distintas maneras de abordar temáticas generales o específicas que forman parte de los cursos introductorios a partir de relatos o fragmentos. Se presenta la temática, el texto literario utilizado en clase y su empleo y finalidad. Por razones de extensión solo se presenta una selección de temáticas y relatos utilizados para su discusión en clase.

\section{Relatos que permiten tener una visión general de los desafíos cognitivos que plantea una investigación social}

En esta sección se abordan tres relatos: La ausencia del Sr. Glass y Los tres instrumentos de la muerte, de Gilbert K. Chesterton (Chesterton, 2008), y Silver Blaze, de Arthur Conan Doyle (Conan Doyle, 1953).

Los protagonistas de estos relatos, a saber, el Padre Brown en las obras de Chesterton y Sherlock Holmes en la de Conan Doyle encarnan prototipos de detective diferentes. Sherlock Holmes es el investigador que se posiciona de forma desapasionada en la escena del crimen y elabora su explicación a partir de la observación y relacionamiento de la evidencia disponible. Encarna la posición inductivista en filosofía de la ciencia en cuanto al énfasis en comenzar el proceso de investigación a partir de la observación. En uno de sus cuentos Holmes afirma4: "Constituye un craso error teorizar sin poseer datos. Uno empieza de manera insensible a retorcer los hechos para acomodarlos a sus hipótesis, en vez de acomodar las hipótesis a los hechos" (Conan Doyle, 1953, pág. 356). Por su parte, el Padre Brown encarna la posición del racionalismo (o quizá del realismo), y elabora teorías primero y luego procede a revisar la evidencia. En uno de los relatos, el Padre Brown describe su método: su punto de partida es comprender la mente del criminal para poder detectar a posteriori quién fue el asesino y 6 : "Planeé todos y cada uno de los crímenes con mucho cuidado - prosiguió el padre Brown-; pensé en cómo llevarlos a cabo, y qué estado de ánimo era necesario para hacerlo. Y cuando estuve seguro de sentir exactamente lo mismo que el asesino, por supuesto, pude saber quién era" (Chesterton, 2008, pág. 720).

Más allá del énfasis empirista o racionalista, las sagas del Padre Brown y de Sherlock Holmes tienen suficiente riqueza literaria como para proveer sutiles escenarios narrativos a partir de los cuales discutir temas de metodología de investigación. En los relatos seleccionados es posible trabajar tanto los componentes de un problema de investigación (pregunta, teoría e hipótesis) como el diseño de estrategias de abordaje y resolución de los problemas de conocimiento, por ejemplo, en lo relacionado al diseño de investigación, elaboración e interpretación de datos. En definitiva, ambos relatos permiten abordar todo lo que habitualmente se define como metodología de investigación (Leal Carretero, 2008).

En la enseñanza de Metodología de Investigación en cursos iniciales los docentes enfrentan dos problemas recurrentes: 
- Una de las grandes dificultades es que el estudiante entienda por qué el principio de una investigación requiere una preparación detenida y un esfuerzo considerable de pensamiento en el planteo adecuado de un problema de investigación.

- El otro problema es poder mostrar cuán difícil es escapar de los sesgos habituales del pensamiento humano (también presentes en el trabajo profesional) (Kahneman, 2012).

En los tres relatos escogidos los investigadores se pierden precisamente porque parten de una idea equivocada de los acontecimientos. Al tratarse de sucesos que motivan la presencia de un detective, hay un supuesto básico que preside las investigaciones y que obstaculiza su esclarecimiento: se parte de la idea de que hubo un crimen y de que hay un criminal. Romper con estas prenociones es uno de los obstáculos más difíciles a enfrentar en investigación social (Bourdieu, Chamboredon \& Passeron, 1975; Chalmers, 1988; Bachelard, 1988).

En La ausencia del Sr. Glass (Chesterton, 2008, págs. 269-287) el problema estriba en la pertinencia de dos teorías alternativas para explicar la evidencia disponible. La viuda MacNab hospeda en su casa a un joven que con el tiempo pretende casarse con su hija. El problema es que se trata de un desconocido y la viuda desconfía: no se sabe qué hace para vivir, se encierra en su habitación y estudia con la puerta cerrada con llave, se oyen voces pero siempre está solo en la habitación. A esto se suma la presencia de un hombre misterioso con sombrero de copa con quien el joven mantuvo una discusión a través de la ventana de su habitación. Ocurre un hecho extraño. La hija de la viuda escucha una fuerte discusión dentro de la habitación del joven entre este y un tal Sr. Glass. Posteriormente, la joven observa por la ventana de la habitación y ve al joven tendido en el piso en una pose extraña. Inmediatamente va a buscar al Padre Brown, quien estaba discutiendo sobre el caso con un criminólogo retirado. El criminólogo acompaña al Padre Brown, fuerzan la puerta de la habitación del joven y lo hallan en el piso maniatado y amordazado, pero vivo. Sin desatarlo, ni sacarle la mordaza, el criminólogo propone una teoría sobre quién es el Sr. Glass, cómo es y qué problema ha tenido con el joven a partir de la interpretación de la evidencia disponible en la habitación. Llega a la conclusión de que el joven ha discutido con el Sr. Glass y que presumiblemente sea el responsable de su desaparición.

La llave del relato está en que la presunción de la existencia de un tal Sr. Glass es equivocada. Por tanto, todas las elucubraciones acerca de una rivalidad entre el joven y el Sr. Glass son falsas. Partiendo de esta idea falsa, el criminólogo interpreta cada objeto fuera de lugar en la habitación como un elemento que fue utilizado en el conflicto entre el presunto Sr. Glass y el joven.

El Padre Brown llega a la conclusión de que el muchacho es un aspirante a mago que también quiere ser malabarista, ventrílocuo y escapista tras seguir atentamente la explicación del criminólogo. En un momento dado percibe que el joven que está amordazado y maniatado se ríe y puede interpretar las evidencias de una manera alternativa que permite resolver las inconsistencias que aparecían en la explicación del criminólogo.

Antes que humillar a su rival, el Padre Brown muestra el valor de pensar a partir de hipótesis rivales. Es precisamente en las inconsistencias de la explicación del criminólogo que el padre Brown encuentra elementos que lo conducen a plantear una explicación alternativa. 
En Los tres instrumentos de la muerte (Chesterton, 2008), el autor plantea una situación extremadamente compleja. El hecho que se busca explicar podría ser un homicidio no intencional, un suicidio o un accidente. Los hechos parecen ser claros. El secretario (Patrick Royce) de un conocido filántropo (Sir Aaron Armstrong) decide asesinar a su empleador (que vive en su casa) porque este se niega a aceptar que se case con su hija (Alice Armstrong). Más aún, Patrick Royce confiesa su crimen y Alice (su pretendida), quien fue testigo de los hechos, confirma el relato de Royce.

La riqueza del relato consiste en que la confesión de Royce y el testimonio de Alice resultan insuficientes para Brown, quien maneja otra teoría.

Sir Aaron Armstrong era un personaje público muy conocido por su hilaridad, buen humor y su profesión de alegría como herramienta para superar los vicios. Brown, conocedor del pasado de Armstrong, sabe que la "religión de la alegría" que este profesa "es una religión cruel" y que "la alegría sin humor puede llegar a ser muy irritante". El pasado alcohólico de Armstrong y su maníaca profesión le hacen pensar a Brown desde un principio que bien podría ser un suicida. Los policías investigadores no manejan esta hipótesis sino que asumen desde un principio que hubo un homicidio.

El caso es complejo porque Patrick Royce forcejea con Armstrong en su habitación para evitar que este se suicide. En medio del forcejeo entra Alice, quien ve a su padre en la ventana y a Royce, que, según observa, quiere atacar a su padre con una cuerda. Alice corta la cuerda, se desmaya y su padre (que estaba atado) cae desde la ventana sin que ella lo vea. Royce asume la responsabilidad del supuesto homicidio para que su prometida no sepa que fue a causa de su participación que el padre cayó de la ventana. La evidencia parece ser concluyente, y la confesión y el testimonio visual parecen dar por cerrado el caso. Pero nada de esto convence al Padre Brown, que maneja una hipótesis alternativa que puede explicar los hechos con mejor ajuste a la evidencia disponible.

Silver Blaze (Conan Doyle, 1953) es un relato en el que la capacidad del investigador para formar hipótesis alternativas también es la clave para la resolución del caso. A diferencia de los otros, la riqueza de este relato está en el minucioso trabajo de reconstrucción de los hechos a partir de la evidencia disponible. David Collier indica que Silver Blaze es un relato perfecto para estudiar las diferentes maneras de procesar la evidencia en un estudio de tipo process tracing (Collier, 2011).

En este relato, Holmes se enfrenta a la desaparición de un célebre caballo de carreras y del presunto asesinato de su entrenador. El caballo está oculto en el establo de los dueños de uno de los rivales de Silver Blaze. El entrenador no fue asesinado, murió accidentalmente cuando pretendía dañar a Silver Blaze con el cuchillo de un cirujano para que no ganase la carrera. En el momento de cometer el fraude una distracción hace que el caballo lo patee y el golpe provoca la muerte del entrenador.

Sobre el final del cuento Holmes reconstruye la manera de resolver el caso. Se trata de un excelente ejemplo de cómo descartar hipótesis y generar nuevas ideas a partir del análisis de la evidencia?.

Los tres relatos pueden trabajarse mediante lectura en clase o pueden fijarse como tarea domiciliaria con un conjunto de preguntas focalizadas en los temas que el docente quiera trabajar. 


\section{Relatos para abordar temáticas específicas}

\section{Análisis tripartito del conocimiento y crítica de E. Gettier}

Uno de los objetivos principales en epistemología es definir conocimiento. Como es sabido, el análisis tripartito del conocimiento -la teoría tradicional del conocimientofue cuestionado en 1963 por el filósofo E. Gettier, quien se replanteó el problema de la definición de conocimiento (Gettier, 1963; Dancy, 1993; Pritchard, 2010).

La definición tripartita asegura que tenemos conocimiento cuando hay creencia verdadera justificada (justified true belief) ${ }^{8}$. A partir de una serie de ejemplos Gettier muestra que esta definición es insuficiente puesto que es posible inferir una creencia verdadera a partir de una creencia cuya justificación es falsa. Por tanto, es posible tener una creencia verdadera justificada y, aun así, no tener conocimiento. A partir de este planteo han surgido varias teorías que intentan proponer una cuarta cláusula que haga a la definición de conocimiento inmune a los ejemplos de Gettier (Dancy, 1993).

Esta discusión es una excelente introducción a un curso de metodología de investigación puesto que plantea en términos analíticos el corazón de los problemas que enfrenta quien quiere conocer. Para que el estudiante entienda la crítica de Gettier debe comprender cabalmente la teoría tripartita del conocimiento.

Para discutir esta temática hemos optado por utilizar el cuento La ventana abierta, de Saki (Munro, 1967). En dicho cuento, un personaje se refugia en el campo para recuperarse de dolencias asociadas al estrés. Su hermana le facilita una tarjeta de presentación para contactarse con vecinos de la zona y así no pasar la temporada en soledad. En la primera casa rural que visita es recibido por una niña, Vera, que le cuenta una historia fantástica.

Vivía con su tía, la cual quedó muy alterada luego de la desaparición de su esposo e hijos tres años atrás en una jornada de caza. La fijación de la tía era dejar la ventana del living abierta porque por allí siempre entraba su esposo al llegar de caza. La tía aún espera que su marido e hijos vuelvan. Llega la tía a la recepción y es presentada al Sr. Nuttel. Cuando la tía le cuenta que su marido e hijos están por regresar, Nuttel no puede menos que sentir lástima por la mujer, así como una gran incomodidad. Cuando marido e hijos (cuya desaparición es una mentira) regresan, el Sr. Nuttel, que está de espaldas a la ventana, observa que la tía los recibe. Cuando gira y los ve entrar sufre un ataque de nervios y se va sin despedirse. La pequeña Vera resulta ser una mentirosa contumaz y al final del cuento inventa una historia sobre Nuttel tan truculenta como la que le relatara a este.

La consigna de la discusión de este relato es responder a la siguiente pregunta: ¿Puede considerarse La ventana abierta como un ejemplo de Gettier? El objetivo de la consigna es que los estudiantes puedan diferenciar un caso en el que se burla uno de los principios del análisis tripartito de un caso que ejemplifica el problema planteado por Gettier. En el cuento de Saki hay una intención deliberada de engañar a una persona, por tanto no constituye un ejemplo de las anomalías señaladas por Gettier.

\section{El problema apariencia y realidad}

Tal como lo plantea Bertrand Russell, el problema de apariencia y realidad aparece cuando queremos conocer la realidad a través de nuestros sentidos (Russell, 1928). Aquello que llega a nuestros sentidos son datos referidos a los objetos externos y estos datos tienen una relación peculiar con dichos objetos, que se ve afectada por un conjunto de factores que hacen a la posición relativa del observador frente al objeto. Por tanto, los datos de los sentidos nos comunican formas y colores aparentes y la labor del investigador consiste en determinar la correspondencia de dichos datos con el objeto real. 
Este problema es el punto de partida para el planteo de la discusión sobre la existencia del mundo exterior a la mente sobre la materia y qué es la materia. A su vez, constituye una pieza clave para la comprensión de las posiciones materialistas e idealistas que aún son corrientes importantes en ciencias sociales, precisamente porque dichas posiciones difieren en la respuesta que dan al problema de la realidad y la materia. Por tanto, es un problema relevante y que el estudiante debe comprender e incorporar en su formación como investigador.

Asimismo, la fábula La paloma sedienta (Esopo, 2013) es un texto literario interesante para poner en práctica elementos de la discusión de apariencia y realidad. En la fábula, una paloma que está buscando agua se precipita sobre un cartel de madera que imita de manera muy vívida una fuente. La paloma choca con el cartel y queda echada en el suelo, donde es capturada.

La fábula de la paloma sedienta se utiliza en ejercicios que plantean a los estudiantes una serie de relatos muy breves y una serie de temas abordados en el curso. El estudiante debe vincular cada cuento con una temática y justificar esta vinculación.

\section{La noción de obstáculo epistemológico}

La noción de obstáculo epistemológico de G. Bachelard (Bachelard, 1988; Bourdieu, Chamboredon \& Passeron, 1975) es una temática que puede ser complementaria del problema de apariencia y realidad.

Según Bachelard, el principal obstáculo en el camino del conocimiento son las ideas previas que posee el investigador, o incluso la comunidad de investigadores. Estas ideas impiden al investigador concebir ciertas posibilidades y explorar caminos alternativos de investigación. Frente a este problema Bachelard plantea la famosa noción de vigilancia epistemológica según la cual la única forma de avanzar es ser consciente y crítico con los supuestos que empleamos en la investigación.

La fábula china traducida con el título La sospecha -recopilada por Bernardo Kordon en una antología de cuentos tradicionales chinos- es un excelente texto para trabajar estos conceptos difíciles para los estudiantes (Kordon, 1976). En esta fábula un campesino pierde un hacha y sospecha que le fue robada por el hijo de su vecino sobre la base de que cuando vio al muchacho este caminaba como un ladrón, miraba como un ladrón, tenía los modos y los aires de un ladrón. Durante el día encuentra el hacha apoyada sobre un árbol que había estado cortando el día anterior. Cuando vuelve a ver al hijo de su vecino este había perdido la apariencia de ladrón.

A partir de este relato se puede discutir sobre la fuerza de los prejuicios, más precisamente sobre cómo juicios previos sobre personas o cosas nos predisponen a ver la realidad a través de un cristal que la deforma. En un episodio relevante del cuento el leñador encuentra su hacha en un lugar donde él podría haberla dejado. Como disparador de la discusión sobre el problema de los supuestos en la observación y cómo ponerlos en cuestión se plantea la pregunta, ¿Qué podría haber sucedido si el leñador no hubiese encontrado el hacha? 


\section{Los problemas de la inferencia, los problemas de investigar}

La definición de inferencia como extraer conocimientos de otros conocimientos refleja la tarea básica de un trabajo de investigación. Los investigadores siempre están produciendo nuevos conocimientos a partir de muy variadas formas de conocer como son la observación, la elaboración de teorías y la intuición. La inducción, la deducción y la abducción son las tres formas inferenciales más trabajadas en los manuales y cursos de metodología de la investigación. Previo a discutir estas formas inferenciales es altamente relevante para el aprendizaje de los estudiantes discutir los problemas generales que enfrentamos al elaborar inferencias.

Para abordar esta discusión se pueden emplear dos relatos provenientes de tradiciones literarias disímiles: la fábula de Esopo titulada El león viejo y el zorro (Esopo, 2013) y Funes el memorioso, de Jorge Luis Borges (Borges, 1998).

La primera es un estupendo micro-relato que permite situar al estudiante frente al gran desafío que todo investigador debe sortear para conocer: el problema de tener que llegar a una conclusión sin poder observar todos los hechos que sería necesario observar para arribar a una conclusión irrefutable.

La fábula se trata de un león viejo que ya no puede cazar y para alimentarse procede a realizar la siguiente treta: haciéndose el enfermo se tiende en una cueva y cuando otros animales van a visitarlo los hace entrar y se los come. El zorro se muestra interesado y desde fuera de la cueva pregunta al león por su estado. El león le dice que está muy mal y que le gustaría verlo. El zorro agradece la invitación pero la rechaza argumentando que ve huellas de entrada pero no de salida.

El relato plantea, de forma abierta y provechosa para la discusión, el clásico problema de confiar en los indicios, muchas veces incompletos y fragmentarios, para arribar al conocimiento de sucesos. El zorro podría confirmar su hipótesis entrando a la cueva y observando si hay restos de animales. Pero si lo hace probablemente sea devorado por el león. De la misma manera, los investigadores o bien tienen limitaciones para observar el objeto de su interés o si lo hacen pueden alterar los resultados que quieren observar. Por tanto, necesitan confiar en indicios indirectos y a veces remotos, y el valor de sus inferencias se establecerá en virtud de la validez y confiabilidad de dichos indicios.

El segundo relato, Funes el memorioso, plantea el mismo problema pero desde un ángulo muy diferente. Como es sabido, la prodigiosa memoria de Funes le permite recordar cada instante experimentado, por ello es imposible para él realizar cualquier clase de generalización: “No solo le costaba comprender que el símbolo genérico perro abarcara tantos individuos dispares de diversos tamaños y diversa forma: le molestaba que el perro de las tres y catorce (visto de perfil) tuviera el mismo nombre que el perro de las tres y cuarto (visto de frente). Su propia cara en el espejo, sus propias manos, le sorprendían cada vez" (Borges, 1998, pág. 134). El mundo de Funes es "multiforme, instantáneo y casi intolerablemente preciso" (Borges, 1998, pág. 134) ${ }^{9}$.

El cuento permite discutir un problema central de las inferencias que habitualmente realizan los investigadores sociales: el problema de la generalización. Permite discutir este problema mostrando con mucha precisión y lucidez el problema de una mente que no puede abstraer, ni generalizar, ni inferir. Muestra, por oposición, la necesidad de abstraer, generalizar e inferir para tener un conocimiento que trascienda el presente y lo banal: “Mi memoria, señor, es como vaciadero de basuras", afirma Funes (Borges, 1998, pág. 131). 
Finalmente, el cuento permite trabajar con los estudiantes las limitaciones propias de las representaciones socio-científicas (Ragin, 2007) pues las representaciones de los cientistas sociales están lejos de la precisión, vividez y detalle de una memoria como la de Funes. Por el contrario, se trata de representaciones limitadas por las interrogantes y los enfoques conceptuales precisos empleados por el investigador. La validez de dichas representaciones se circunscribe a las interrogantes y a los enfoques empleados.

\section{Los conceptos de validez y confiabilidad}

En ciencias sociales medir implica observar el funcionamiento de ciertos conceptos teóricos (y sus relaciones) en la vida social. Esta observación se logra luego de un proceso conocido como operacionalización, el cual implica ir desde una definición conceptual hacia indicadores empíricos concretos del concepto. Una pregunta que surge a cualquier investigador es si ha realizado correctamente la operacionalización de los conceptos que desea medir. En el plano metodológico esta pregunta refiere a las bondades de una buena medida pues un proceso o instrumento de medición debe tener dos cualidades básicas: confiabilidad y validez.

La confiabilidad es la magnitud o la medida en que un instrumento o procedimiento de medición arroja similares resultados en repetidas mediciones. Confiable es un indicador que tiende a la consistencia en sucesivas mediciones (es decir, tiende a obtener valores similares siendo que entre medición y medición no han ocurrido hechos que ameriten o justifiquen una variación importante).

La validez refiere a una cualidad diferente. Un indicador de un concepto abstracto se considera válido en tanto sea capaz de medir dicho concepto y no otro. La validez tiene que ver, entonces, con la relación entre indicador y concepto.

El principal obstáculo que presenta la enseñanza de estos conceptos proviene de la escasa precisión que es requerida en la vida cotidiana para dar a entender a otro qué es lo que queremos o qué es lo que necesitamos. Múltiples aspectos contextuales de una interacción nos permiten comprender una solicitud inespecífica o imprecisa. Pero cuando queremos medir conceptos a través de una encuesta el investigador no forma parte del contexto de interacción en el que se aplica la encuesta.

Para trabajar los conceptos de validez y confiabilidad puede utilizarse el cuento El papel pintado. Se trata de un relato oral llevado a la escritura por Jean Claude Carrière ${ }^{10}$ (Carrière, 2001, pág. 316). En este relato breve, ambientado en una ciudad francesa no especificada, el personaje es un nuevo inquilino en un edificio de apartamentos. Es un hombre simpático que dedica una jornada a visitar a sus nuevos vecinos. El vecino del apartamento que está encima del suyo lo invita a pasar y le ofrece coñac. El empapelado, que cubre todo el apartamento, le resulta encantador. Entusiasmado con emplear el mismo empapelado para su apartamento pregunta a su vecino cuántos rollos ha comprado. El vecino le dice que 28. El nuevo inquilino compra los 28 rollos y cubre todo el apartamento que es exactamente igual al de su vecino. Cuando termina le sobran 10 rollos. Extrañado, vuelve a visitar al vecino de arriba y le plantea la situación: compró 28 rollos, forró todo el apartamento y le sobraron 10. "A mí también", responde su vecino.

El nuevo inquilino quería saber cuántos rollos usó (concepto) pero preguntó cuántos rollos compró (indicador). La pregunta genera una respuesta confiable, en la medida en que no presenta problemas de comprensión y presumiblemente arrojará los mismos resultados en repetidos intentos, pero no es válida porque no representa el concepto cuyo valor se quiere establecer. 


\section{¿Qué es una hipótesis? ¿Cómo funciona el pensamiento hipotético?}

La elaboración de una hipótesis es una herramienta que los investigadores emplean en múltiples instancias en una investigación. Se considera el punto de partida de una investigación por la tradición hipotético-deductiva (Chalmers, 1988; Elster, 2010) o como una herramienta para la elaboración de teorías por la tradición inductiva (Neumann, 1997; Timmermans \& Tavory, 2012). En investigación se recurre a hipótesis de forma sistemática para organizar los materiales a través de los cuales pensamos la realidad.

Enseñar qué es una hipótesis presenta el mismo problema que hemos referido para otros conceptos metodológicos: es fácil comprender la definición pero muy difícil aprender cómo elaborar hipótesis. A este respecto, los relatos permiten que el estudiante visualice el pensamiento hipotético en acción. De esa manera, puede incorporar un elemento dinámico difícil de generar con el estudio de una definición y con la elaboración de ejemplos. Asimismo, los relatos permiten mostrar que el razonamiento hipotético no es ajeno al pensamiento cotidiano de cualquier persona.

Un caso excelente para este tema es otra fábula china recopilada por Bernardo Kordon titulada Palillos de marfil. En esta fábula, el príncipe Ki (tío y consejero del rey) elabora una cadena de hipótesis a partir de un hecho aparentemente nimio. Esta cadena de hipótesis predice un desenlace negativo para el reinado de su sobrino, Chu (último rey de la dinastía Chang según se informa en la fábula). El suceso que desencadena la serie de hipótesis es la orden de Chu de que se le fabricasen palillos de marfil para comer. A partir de este hecho aparentemente intrascendente, el príncipe Ki razona:

"Los palillos de marfil no pueden usarse con tazones y platos de barro cocido, exigen vasos tallados en cuernos de rinoceronte y platos de jade, donde en vez de cereales y legumbres deben servirse manjares exquisitos, como ser colas de elefante y fetos de tigre. Llegado a esto, difícilmente el rey estaría dispuesto a vestir telas burdas y vivir bajo un techo de paja: encargaría sedas y mansiones lujosas (Kordon, 1976, pág. 17)".

A partir de este encadenamiento de hipótesis Ki concluye: "Me inquieta adónde conducirá todo esto" (Kordon, 1976, pág. 17). Con el tiempo, Chu asola el reino para financiar sus lujos así como tortura a los súbditos que se oponen.

La clave de la discusión en clase es que los estudiantes puedan reconstruir el punto de partida y conectar la serie de pasos que culmina en la hipótesis final de que el futuro del reino es inquietante. En el punto de partida hay una simple orden (elaborar palillos de marfil para comer), pero cabe preguntarse, ¿qué justifica el razonamiento que hace Ki? ¿Es mera casualidad el acierto? ¿Qué permite conectar a los palillos de marfil con la hipótesis que anuncia un futuro inquietante?

A partir de la discusión de esta fábula el docente puede trabajar la conexión teoría- hipótesis, así como la manera de conectar teoría y realidad a través de una hipótesis o un conjunto de hipótesis ${ }^{11}$. También puede trabajar ideas complejas, como el enfoque sistémico en ciencias sociales. En este sentido lo interesante del relato es mostrar cómo es posible predecir transformaciones en la economía y una crisis política a partir de un pequeño cambio en las costumbres (esfera de la cultura). La teoría que permite hacer esta predicción concibe la sociedad como un todo integrado por diferentes componentes altamente relacionados entre sí, de manera tal que un cambio en uno de dichos componentes puede acarrear cambios importantes en los otros y es conocida como enfoque sistémico (Bunge, 2001). 


\section{Dinámicas de clase. ¿Cómo emplear los textos literarios para promover la discusión de temas epistemológicos y metodológicos?}

Los textos y conceptos aquí señalados se utilizaron en el marco de cursos de grado de Introducción a la Investigación, uno con foco en fundamentos epistemológicos de la investigación científica y construcción de problemas de investigación y otro centrado en diseño y técnicas de investigación.

Dado el carácter introductorio de estos cursos y la consecuente escasa familiaridad de los estudiantes con los conceptos trabajados, la inclusión de obras literarias suele resultar de utilidad para oficiar como puente entre contenidos conocidos o fácilmente accesibles a los estudiantes y contenidos más distantes y de mayor abstracción.

Este recurso también reporta ventajas en términos de vinculación y desarrollo de conceptos a lo largo del curso. En tanto que el concepto queda anclado a un relato, se vuelve un recurso memorístico de fácil acceso durante el transcurso del curso y permite aludir a ese relato para retomar la idea y vincularla con nuevos conceptos.

El desarrollo de un trabajo reflexivo en torno a los contenidos de las narraciones utilizadas es lo que permite transitar desde un ejemplo específico hacia conceptos más generales. Esto puede tomar diferentes formas dependiendo de los conceptos a trabajar y de las características del relato que se escojan para introducirlos. El uso de narraciones, relatos breves o fábulas puede apoyar tanto la tarea en el aula como fuera de ella y puede utilizarse para introducir ideas nuevas o reforzar nociones ya trabajadas. También puede ser una herramienta de utilidad para generar evaluaciones en las que los estudiantes deban aplicar lo aprendido a un contexto concreto, debiendo demostrar no solo el entendimiento del concepto en cuestión sino también su manejo práctico.

A continuación se presentarán tres tipos de dinámicas usualmente empleadas:

- Identificar conceptos o discusiones;

- Identificar un argumento en un texto literario y elaborar ideas a partir de él;

- Experimentar problemas que enfrenta un investigador en su trabajo.

\section{Identificar conceptos o discusiones}

El reconocimiento de conceptos y su correcta aplicación es parte relevante del aprendizaje del estudiante. Los textos literarios brindan la posibilidad de identificar posiciones teóricas o problemas intelectuales. Esto exige un esfuerzo para el estudiante, quien debe tener claro el concepto o problema teórico como para poder identificarlo en un texto donde dicho concepto o problema aparece de manera implícita (Cottrell, 2005).

Cualquiera de los textos literarios anteriormente presentados puede utilizarse con este fin. Para ello es aconsejable, primero, trabajar las temáticas a partir de bibliografía tradicional, y, luego, solicitar al estudiante que analice el texto literario en cuestión para identificar o precisar los conceptos o problemas de conocimiento abordados en esos textos.

Identificar un argumento en un texto literario y elaborar ideas a partir de él Poder identificar argumentos es una habilidad clave en estudiantes de investigación social, ya que en el futuro dedicarán buena parte de sus carreras profesionales a construir y criticar argumentos. La clave de esta dinámica es que el estudiante separe aquello que constituye parte de un argumento (usar razones para dar soporte a un punto de vista con intención de persuadir a una audiencia) de aquello que no. Nuevamente, los textos literarios ofrecen 
una oportunidad única ya que no están elaborados con la intención de argumentar una posición sino que los personajes simplemente argumentan en forma natural a lo largo de la historia. El estudiante debe esforzarse por captar la esencia de un argumento que no es presentado de forma explícita como en un trabajo científico.

Varios de los textos literarios presentados anteriormente pueden utilizarse con este fin. Mediante una guía de preguntas adecuada es posible orientar al estudiante para que logre formalizar un argumento teórico a partir de una situación concreta (presentada en el relato literario) para luego aplicarlo a situaciones concretas diferentes a la presentada en el relato. De este modo se lo estimula a ejercitar tanto la capacidad de abstraer conceptos generales a partir de una idea particular -apelando una lógica inductiva- como a aplicar estos conceptos a fenómenos sociales concretos -apelando a una lógica deductiva-. Finalmente, se lo exhorta a vincular el argumento elaborado con conceptos ya trabajados previamente.

\section{Experimentar problemas que enfrenta un investigador en su trabajo}

Una dinámica que se puede emplear para que el estudiante experimente los problemas de un investigador es la de realizar una representación teatral en clase. A modo de ejemplo, para este fin la obra breve Reportaje 1 de Leo Maslíah es altamente recomendable (Maslíah, 2007). A partir de la representación de dicha obra es posible trabajar sobre las dificultades que presenta la interacción en una entrevista. El texto muestra a un periodista improvisado que consulta a una persona pública para realizar una entrevista. Contra la expectativa del solicitante, el personaje acepta la entrevista y le pide que comience. El entrevistador no cuenta con una guía de entrevista e improvisa y al hacerlo no es capaz de formular una pregunta con claridad, hasta que provoca la ira del entrevistado.

En el cuento En el bosque de Ryunosuke Akutagawa, se presentan las declaraciones de una serie de testigos e involucrados en la muerte de Takejiro Kanazawa (Akutagawa, 1977, pág. 40). A partir de la lectura de las sucesivas declaraciones se conforma un panorama de versiones contrapuestas acerca de un mismo hecho. Este cuento, originalmente concebido para ilustrar la mentalidad tradicional japonesa, sirve para ilustrar las dificultades a las que se enfrenta un investigador cuando se aproxima a un fenómeno a partir de los discursos de los sujetos involucrados.

Tras la lectura del cuento, en el contexto de clase, se solicita a los estudiantes que discutan en pequeños subgrupos cuál es la dificultad que se presenta en el cuento para resolver las circunstancias de la muerte de Takejiro Kanazawa y cómo resolverían esa dificultad. Luego, con la guía del docente se realiza una puesta a punto que implica que representantes de los distintos subgrupos discutan y argumenten sus conclusiones. Un elemento adicional que fortalece los aprendizajes de los estudiantes es mostrar casos reales que siguen el patrón detectado en En el bosque. En nuestro caso se trabaja con artículos de prensa que informan sobre casos judiciales con relatos contradictorios entre los partícipes del hecho.

\section{Evaluación del uso de textos literarios en clase}

Como insumo para conocer la valoración de los estudiantes sobre la utilidad del uso de textos literarios en las dinámicas de clase se cuenta con los resultados de una encuesta aplicada a estudiantes de cursos de metodología en los que se utilizó esta herramienta. Estos datos son de carácter exploratorio y no permiten evaluar resultados de aprendizaje. La información analizada surge de un cuestionario aplicado a estudiantes que participaron en tres cursos metodológicos impartidos en las licenciaturas de Estudios Internacionales (EI) y de Administración (LA) de la Universidad ORT Uruguay. Los cursos son los siguientes: Fundamentos de la Investigación Social, impartido en el primer semestre de El, Métodos de Investigación, correspondiente al tercer semestre de El, y Taller de investigación aplicada, del segundo semestre de LA. 
La encuesta fue realizada al final de los cursos dictados en 2016 y 2017 (primer semestre del año), y cuenta con un total de 141 respondentes. El cuestionario incluía preguntas de evaluación del curso, evaluación del uso de textos literarios y evaluación de las dinámicas empleadas en clase. Las encuestas fueron respondidas por aproximadamente el 75\% de los estudiantes de cada curso.

Las preguntas del formulario se orientaron a medir la motivación que generaba en los estudiantes el uso de textos literarios, su aporte a la comprensión de los temas tratados y su valor de cara a la elaboración del trabajo final del curso. Complementariamente, se evaluaron las dinámicas propuestas para el trabajo con estos textos con relación a si se usaron en su justa medida y si contaron con consignas claras para su uso.

Los estudiantes mostraron una evaluación favorable del empleo de textos literarios: el $45 \%$ evaluó el uso de estos textos como motivadores o muy motivadores (valores 6 y 7 en una escala del 1 al 7) y el 51\% expresó que les resultó valioso o muy valioso para la comprensión de los temas abordados. Algo menos favorable fue la valoración de la utilidad de estos textos de cara a la ejecución del trabajo final del curso.

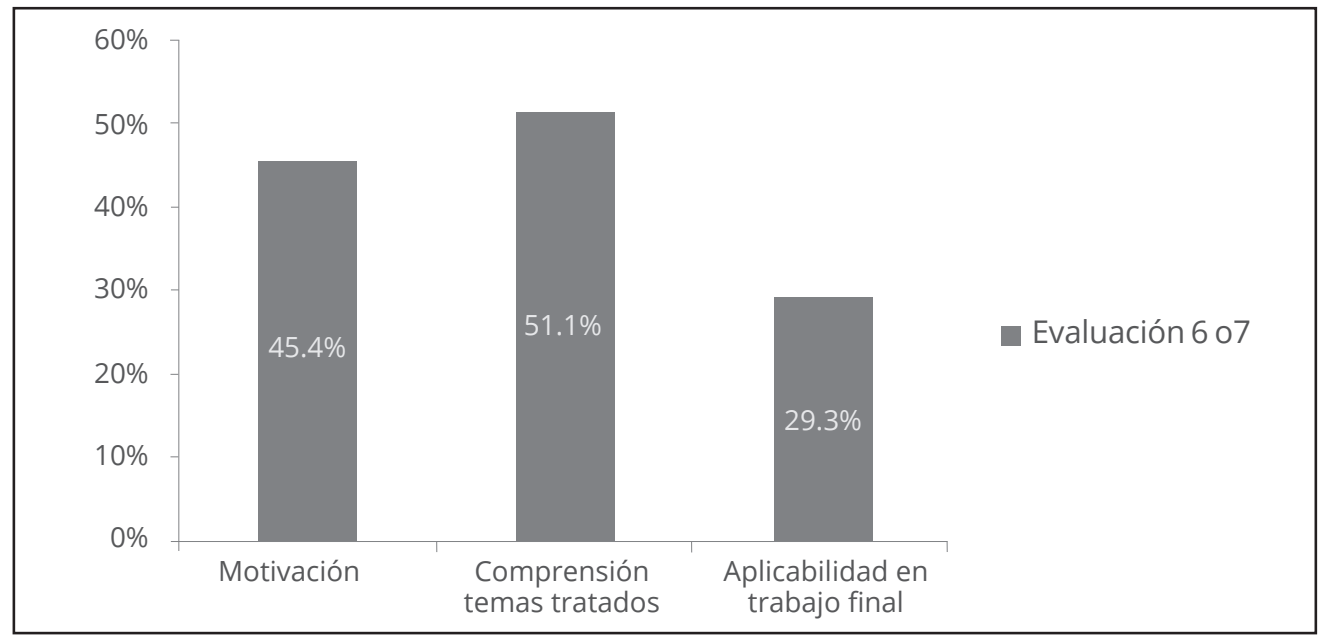

Gráfico 1. Porcentaje de respuestas favorables o muy favorables (valores 6 y 7 de la escala) acerca de la motivación, la ayuda a la comprensión de los temas y la aplicabilidad al trabajo final del curso, con respecto al uso de textos literarios en clase (Escala del 1 al 7)

Para cada uno los tres ítems evaluados (motivación, comprensión de los temas tratados y aplicabilidad en el trabajo final), la valoración de los estudiantes tuvo valores promedio por encima del valor medio de la escala (4) con una dispersión baja en torno a la media. 
Tabla 1. Valoración promedio del uso de textos literarios en clase en lo referente a la motivación, la ayuda a la comprensión de los temas y la aplicabilidad al trabajo final del curso (Escala del 1 al 7)

\begin{tabular}{|l|c|c|c|c|c|}
\hline \multicolumn{2}{|c|}{ N N } & Mínimo & Máximo & Mediás & Desv. Típ. \\
\hline $\begin{array}{l}\text { ¿Cuán motivadores han resultado los } \\
\text { Cuentos para involucrarte en los temas } \\
\text { tratados con ellos? }\end{array}$ & 141 & 2 & 7 & 5,34 & 1,281 \\
\hline $\begin{array}{l}\text { ¿Cuán valiosa te resultó esta actividad para } \\
\text { la comprensión de los temas tratados? }\end{array}$ & 141 & 2 & 7 & 5,39 & 1,223 \\
\hline $\begin{array}{l}\text { ¿Cuán valiosa te resultó esta actividad para } \\
\text { la aplicación práctica de los temas tratados } \\
\text { en el trabajo final? }\end{array}$ & 140 & 1 & 7 & 4,53 & 1,510 \\
\hline
\end{tabular}

Las evaluaciones realizadas por los estudiantes presentan variaciones según el semestre en el que se encuentran ${ }^{12}$. Los estudiantes de los semestres superiores tienen una mejor evaluación del uso de textos literarios que los de los semestres iniciales. Esto tiende a mantenerse en los diferentes ítems evaluados.

Tabla 2. Porcentaje de respuestas favorables o muy favorables (valores 6 y 7 de la escala del 1 al 7) acerca de la motivación, la ayuda a la comprensión de los temas y la aplicabilidad al trabajo final del curso según materia cursada y valoración global del dictado del curso (evaluado en una escala del 1 al 7)

\begin{tabular}{|l|c|c|c|c|}
\hline & & \multicolumn{3}{|c|}{ Materia cursada } \\
\hline $\begin{array}{l}\text { Respuestas } \\
\text { favorables o muy } \\
\text { favorables (puntaje } \\
\text { 6 o 7) acerca de: }\end{array}$ & Total & $\begin{array}{c}\text { Fundamentos de } \\
\text { la investigación } \\
\text { (semestre I) }\end{array}$ & $\begin{array}{c}\text { Taller de } \\
\text { investigación } \\
\text { aplicada (entre } \\
\text { semestre II y III) }\end{array}$ & $\begin{array}{c}\text { Métodos de } \\
\text { investigación } \\
\text { (semestre V) }\end{array}$ \\
\hline Motivación & $45,4 \%$ & $38,2 \%$ & $40,6 \%$ & $61,0 \%$ \\
\hline $\begin{array}{l}\text { Comprensión de } \\
\text { temas tratados }\end{array}$ & $51,1 \%$ & $39,7 \%$ & $62,5 \%$ & $61,0 \%$ \\
\hline $\begin{array}{l}\text { Aplicabilidad al } \\
\text { trabajo final }\end{array}$ & $29,3 \%$ & $19,1 \%$ & $37,5 \%$ & $40,0 \%$ \\
\hline
\end{tabular}

Consistentemente con los resultados anteriores, consultados acerca de si recomendarían el uso de esta herramienta, la mayoría de los estudiantes $(61,2 \%)$ declaró que la recomendaría totalmente y un 35,3\% que tal vez la recomendaría, resultando marginal la proporción de quienes no la recomendarían (3,6\%). 


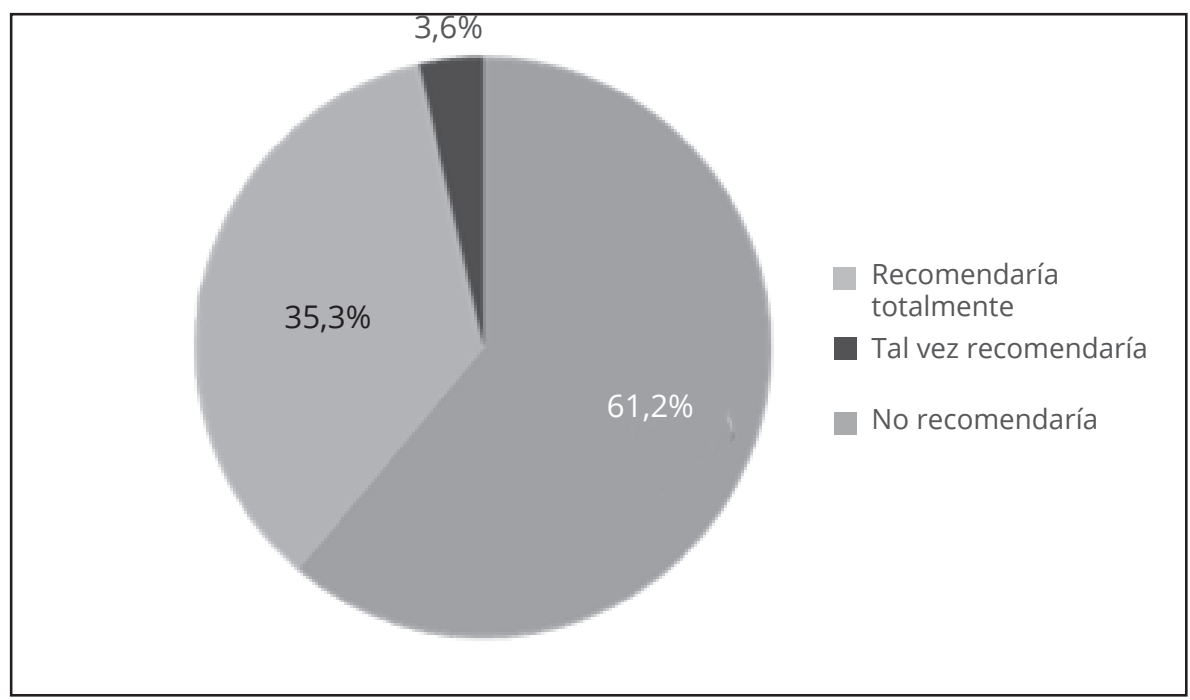

Gráfico 2. Distribución porcentual de los estudiantes según si recomendarían el uso de textos literarios en futuros cursos

Acerca de la forma en que los textos literarios fueron empleados en clase, los estudiantes también manifiestan una opinión favorable: el 81,8\% considera que los textos fueron usados en su justa medida mientras que el 11,7\% hubiera preferido que se utilizaran con más frecuencia. Para la mayoría de los estudiantes (87\%) las pautas brindadas en clase para el trabajo con este tipo de materiales resultaron claras.

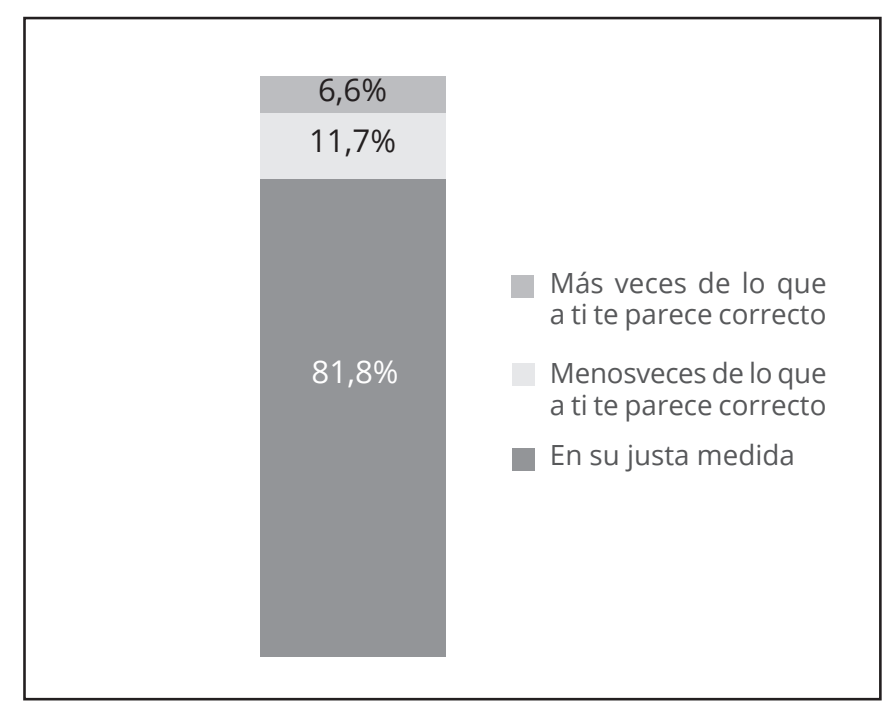

Gráfico 3. Distribución porcentual de los estudiantes según si consideran que el uso de textos literarios se empleó en su justa medida, más veces de lo que creen correcto o menos veces de lo que creen correcto 


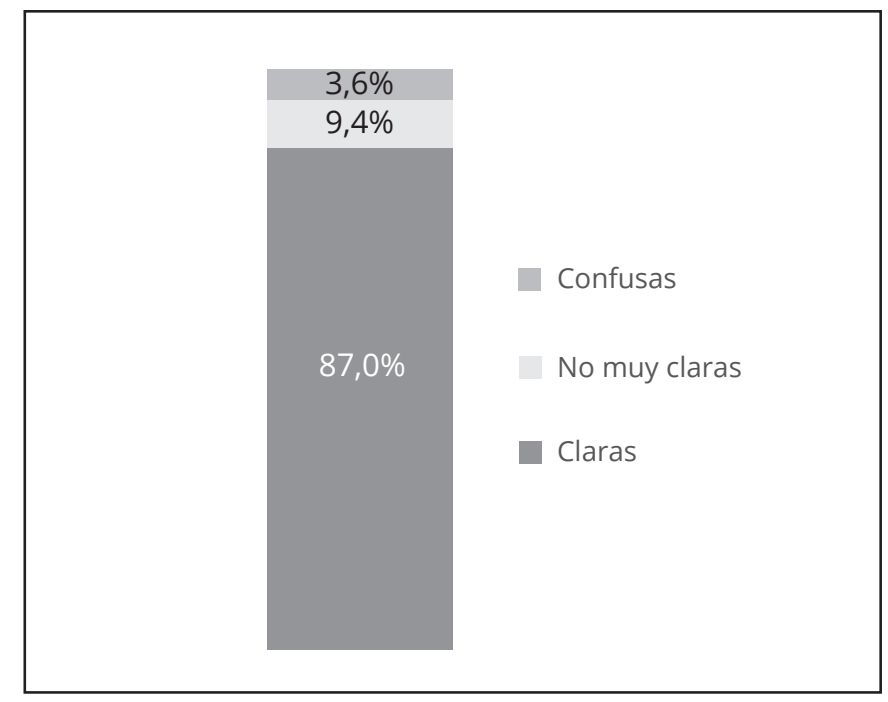

Gráfico 4. Distribución porcentual de los estudiantes según si consideran que las instrucciones brindadas por el profesor para el uso de los textos fueron claras, no muy claras o confusas

\section{Reflexiones Finales}

Este artículo resume la experiencia de trabajo con textos literarios en el marco de cursos introductorios a la epistemología y metodología de las ciencias sociales.

Los relatos aquí presentados permiten tanto problematizar el proceso de investigación en su conjunto (aspecto desarrollado en el apartado A. del capítulo II) como abordar algunas temáticas específicas que suelen tratarse en los cursos vinculados a la materia (aspecto tratado en el apartado B. del capítulo II).

El primer conjunto de relatos, vinculados al proceso de investigación en su conjunto, pueden aportar tanto a la apertura como al cierre de un curso. Incorporados en las primeras clases pueden utilizarse como insumos para problematizar los conocimientos previos con los que los estudiantes llegan al curso. Trabajados sobre el final del curso resultan de utilidad para poner a prueba o profundizar en la comprensión y aplicación de los contenidos ya trabajados.

El segundo conjunto de textos literarios, centrados en temáticas específicas, también pueden utilizarse para introducir el tema o para profundizar en su comprensión. En el apartado III de este trabajo se desarrollaron algunos ejemplos de dinámicas empleadas con algunos de estos textos.

Tanto las temáticas seleccionadas como los textos literarios y las propuestas de trabajo con relación a estos son de carácter abierto, y debieron ser adaptados a los propósitos del curso en cuestión y a las características de los estudiantes. No obstante, el uso de estos materiales requiere ser acompañado de una guía de trabajo que oriente a los estudiantes, tanto para comprender el relato en cuestión (elemento que es preciso asegurar antes de avanzar en su interpretación) como para vincular la narración con los conceptos metodológicos o epistemológicos que se procura trabajar. 
Desde la perspectiva del trabajo docente, la incorporación de estos materiales exige un esfuerzo de preparación que incluye la selección de los textos, la planificación de las dinámicas de clase donde trabajarlos y la corrección de las producciones escritas que emergen del análisis. Esta metodología de trabajo también reporta beneficios en la organización del curso. Una vez trabajados, los relatos literarios se vuelven un recurso fácilmente accesible para retomar en instancias posteriores. A su vez, las discusiones que se generan a partir de la presentación de este tipo de materiales -que implican que los estudiantes brinden interpretaciones preliminares- constituyen una instancia privilegiada para comprender los conocimientos y preconceptos que manejan ${ }^{13}$.

A partir de encuestas aplicadas en la última clase del curso, los resultados de la incorporación de estos materiales en el dictado de las clases en términos de satisfacción estudiantil muestran una valoración positiva de la práctica, tanto a efectos de motivar a los estudiantes a seguir el curso como a efectos de facilitar la comprensión de los temas trabajados. Los datos muestran que el uso de textos literarios es más valorado en los estudiantes de semestres superiores. Este es un tema a monitorear en sucesivas mediciones.

Las valoraciones y la experiencia con los estudiantes en el aula refuerzan la idea de que el uso de estos materiales puede resultar de utilidad para tejer puentes entre los conocimientos previos de los estudiantes y los problemas de conocimiento que se procuró introducir en el marco de estos cursos.

\section{Bibliografía}

Akutagawa, R. (1977). Cuentos. Montevideo: Club del Libro.

Ambrose, S. A., Bridges, M. W., DiPietro, M., Lovett, M. C. \& Norman, M. K. (2010). How learning works. Seven research-based principles for smart teaching. San Francisco: Jossey-Bass.

Bachelard, G. (1988). La formación del espíritu científico. México: Siglo XXI.

Borges, J. L. (1998). Ficciones. Madrid: Alianza.

Bourdieu, P., Chamboredon, J. C. \& Passeron, J. C. (1975). El oficio de sociólogo. Buenos Aires: Siglo XXI.

Bunge, M. (2001). Construyendo puentes entre las ciencias sociales. En Desigualdad y globalización. Cinco conferencias. (págs. 47-74). Buenos Aires: FCS (UBA) - Manantial.

Carrière, J-C. (2001). El círculo de los mentirosos. Barcelona: Debolsillo.

Castellano, U., DeAngelis, J. \& Clark-Ibáñez, M. (2008). Cultivating a sociological perspective using nontraditional texts. Teaching Sociology, 36, 240-253.

Chalmers, A. (1988). ¿Qué es esa cosa llamada ciencia? México: Siglo XXI.

Chesterton, G. K. (2008). Los relatos del Padre Brown. Barcelona: Acantilado.

Collier, D. (2011). Understanding process tracing. PS: Political Science and Politics, 44(4), 823-830. 
Conan Doyle, A. (1953). Sherlock Holmes. Obras completas. Tomo I. Madrid: Aguilar.

Coser, L. (1972). Sociology through literature. Englewood Cliffs: Prentice Hall.

Cottrell, S. (2005). Critical thinking skills. Developing effective analysis and argument. London: Palgrave Study Skills.

Dancy, J. (1993). Introducción a la epistemología contemporánea. Madrid: Tecnos.

Davini, M. C. (2008). Métodos de enseñanza. Didáctica general para maestros y profesores. Buenos Aires: Santillana.

Einstein, A. \& Infeld, L. (1986). La fisica. Aventura del pensamiento. Buenos Aires: Losada.

Elster, J. (2010). La explicación del comportamiento social. Más tuercas y tornillos para las ciencias sociales. Barcelona: Gedisa.

Esopo. (2013). Fábulas. Barcelona: Penguin Clásicos.

Gettier, E. (1963). Is Justified True Belief Knowledge? Analysis, Vol. 23, No. 6. (Jun., 1963), pp. 23(6), 121-123.

Graves, R. (2003). Las aventuras del Sargento Lamb. Barcelona: Edhasa.

Hegtvedt, K. (1991). Teaching sociology of literature through literature. Teaching sociology, 19(1), 1-12.

Hill, M. R. (1987). Novels, Thought Experiments, and Humanist Sociology in the Classroom: Mari Sandoz and "Capital City". Teaching Sociology, 15(1), 38-44.

Jasso, G. (1988). Principles of theoretical analysis. Sociological Theory, 6(1), 1-20.

Jones, R. A. (1975). The use of literature in teaching introductory sociology: a case study. Teaching sociology, 2(2), 177-196.

Kahneman, D. (2012). Pensar rápido, pensar despacio. Buenos Aires: Debate.

Kordon, B. (1976). Así escriben los chinos. Desde la tradición oral hasta nuestros días. Buenos Aires: Orión.

Krause, M. (2003). La economía explicada a mis hijos. Buenos Aires: Aguilar.

Laz, C. (1996). Science fiction and introductory sociology: The "Handmaid" in the Classroom. Teaching Sociology, 24(1), 54-63.

Leal Carretero, F. (2008). Los modelos en las ciencias sociales. En F. Leal Carretero (coord.), Cómo se hacen las ciencias sociales: Una antología de ejemplos y preceptos en homenaje a Fernando Pozos Ponce (págs. 347-411). Guadalajara: Universidad de Guadalajara.

Maslíah, L. (2007). Tres idiotas en busca de una imbécil y otras 30 piezas breves. Buenos Aires: De la Flor. 
Mendizábal, N. (2006). Los componentes del diseño flexible en la investigación cualitativa. En I. Vasilachis, Estrategias de investigación cualitativa. Barcelona: Gedisa.

Munro, H. H. (1967). Los increíbles cuentos de Saki. 31 relatos. Ciudad de México: Novaro.

Neumann, W. L. (1997). Social research methods. Qualitative and quantitative approaches. London: Routledge.

Pritchard, D. (2010). What is this thing called knowledge? (2nd ed.). London: Routledge.

Quivy, R. \& Van Campenhoudt, L. (2001). Manual de investigación en ciencias sociales. México: Limusa.

Ragin, C. (2007). La construcción de la investigación social. Bogotá: Universidad de Los Andes - SAGE.

Russell, B. (1928). Los problemas de la filosofía. Barcelona: Labor.

Sanders, W. B. (ed.) (1976). The sociologist as detective; an introduction to research methods. (Second ed.). New York: Praeger.

Sloman, S. \& Fernbach, P. (2017). The knowledge illusion: why we never think alone. New York: Riverhead Books.

Somerset Maugham, W. (1961). En un biombo chino. Barcelona: Plaza \& Janés.

Sullivan, T. (1982). Introductory Sociology through Literature. Teaching sociology, 10(1), 109-116.

Timmermans, S. \& Tavory, I. (2012). Theory Construction in Qualitative Research: From Grounded Theory to Abductive Analysis. Sociological Theory, 30(3), 167-186.

Twain, M. (2013). El elefante blanco robado y otros cuentos. Ciudad de México: Barataria.

\section{(Endnotes)}

1 Una versión preliminar de este artículo fue presentada en el 2016, en el V Encuentro Latinoamericano de Metodología de las Ciencias Sociales. Para la presente publicación se depuró el contenido de esa primer versión y se incorporó un nuevo apartado con los resultados empíricos, basados en una encuesta de satisfacción a estudiantes participantes de cursos donde se emplearon textos literarios como herramientas para la enseñanza de metodología.

${ }^{2}$ La idea ha llegado a otros campos disciplinares. Es notable el trabajo de Martín Krause en Economía (Krause, 2003).

3 “El principal problema en la introducción a la sociología es la enorme brecha entre los conceptos sociológicos abstractos (e.g. "desviación", "alienación", "sistema" y, por supuesto, "perspectiva sociológica" en sí) y el conjunto de experiencias reales subjetivas -y sin embargo no menos "sociales"del estudiante mismo. Tal como sugieren las respuestas, la literatura ofrece un tercer nivel intermedio de comprensión: describe experiencias que están "fuera" del estudiante y no las suyas propias, lo que le permite enfatizar y generalizar más allá de su conciencia personal. (Jones, 1975, pág. 195)." Traducción libre. 
${ }^{4}$ Se trata de Aventuras de un escándalo en Bohemia.

${ }^{5}$ Se trata de El secreto del Padre Brown.

${ }^{6}$ La perspectiva del Padre Brown tiene muchos puntos de contacto con la sociología comprensiva de Max Weber.

${ }^{7}$ Para profundizar ver (Collier, 2011) y (Sanders (ed.), 1976).

${ }^{8}$ En términos más precisos: “La definición tripartita tiene atractivos obvios. Normalmente, se considera que la primera cláusula, la que dice que, si a sabe que $p$, $p$ es verdadera (que puede leerse como Sap — p), es estipulativa. La segunda cláusula, que, si a sabe que p, a cree que p (Sap — Cap), parece ser de contenido mínimo. La tercera, que, si a sabe que $p$, su creencia de que p está justificada (Sap — CJap), trata de evitar que cuente como conocimiento cualquier acierto casual por parte de un imprudente que confíe acríticamente en sus propias decisiones. Sin embargo, es importante que tengamos en cuenta una consecuencia de esta justificación de la cláusula 3: normalmente una creencia no está justificada por el mero hecho de ser verdadera, de otro modo la cláusula 3 sería redundante" (Dancy, 1993, pág. 39).

${ }^{9}$ Se ha descubierto recientemente que la condición de Funes existe y se le ha llamado hyperthymesia o highly superior autobiographical memory (Sloman \& Fernbach, 2017).

${ }^{10}$ Reconocido guionista francés nacido en 1931. Mantuvo una larga colaboración con Luis Buñuel en Diario de una camarera (1964), Belle de jour (1967), La vía láctea (1969), El discreto encanto de la burguesía (1972), El fantasma de la libertad (1974) y Ese oscuro objeto del deseo (1977).

${ }^{11}$ En este caso la hipótesis no es un acierto casual, tiene un fundamento en una teoría social no explicitada pero que es posible deducir a partir de la hipótesis (aunque no en forma completa, obviamente). Las discusiones y los intentos de formalización por parte de los estudiantes son muy enriquecedores.

${ }^{12}$ Las pruebas de Chi2 para las tablas de contingencia entre Materia cursada y cada uno de los tres ítems evaluados permite rechazar la independencia estadística con un $90 \%$ de confianza.

${ }^{13}$ El conocimiento de las ideas de los estudiantes es un elemento de diagnóstico básico para la planificación de un curso universitario (Ambrose, Bridges, DiPietro, Lovett \& Norman, 2010). 